\title{
Detección histológica y molecular de Helicobacter pylori y genotipificación con base en los genes de virulencia babA2 e ice $A$ en pacientes con patología gástrica benigna
}

\author{
M. Camila Montealegre O., Carlos Alberto Jaramillo H., Gustavo Montealegre L., \\ Giovanna Parra G., M. Magdalena Echeverry de P. y M. del Pilar Delgado P.
}

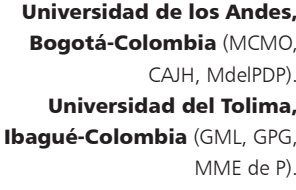

MCMO trabaja actualmente en el Centro Internacional de Entrenamiento e Investigaciones Médicas.

Institución donde se realizó el trabajo: Laboratorio de Diagnóstico Molecular y Bioinformática, Universidad de los Andes. Carrera $1^{\text {a }} \mathrm{N}^{\mathrm{O}}$ 18A-10, Bogotá, DC., Colombia.

Conflicto de interés. Los autores manifiestan que no existe conflicto de interés alguno.

Recibido: 16 de junio de 2009 Aceptado: 5 de octubre de 2009

Correspondencia a:

M. del Pilar Delgado P. mdelgado@uniandes.edu.co.

\section{Introducción}

$H$ elicobacter pylori es un bacilo gramnegativo microaerófilo que coloniza de manera persistente la mucosa gástrica de humanos ${ }^{1,2}$. La infección con esta bacteria ocurre en todo el mundo y la prevalencia varía según la región geográfica, lo que se ha asociado con el nivel socio-económico de la población ${ }^{3}$. En los países en vías de desarrollo, la prevalencia de la infección alcanza $\sim 80 \%$ y la mayoría de las personas adquiere la infección a temprana edad ${ }^{4,5}$.

El curso clínico de la infección es altamente variable y depende de las circunstancias ambientales, factores del hospedero, así como de factores de virulencia de la bacteria, los cuales pueden llegar a ser importantes en el desarrollo diferencial de las patologías ${ }^{3,6,7}$. Inicialmente la infección aguda puede inducir gastritis crónica no atrófica que eventualmente progresa hacia gastritis crónica atrófica, úlcera péptica, metaplasia intestinal, displasia y carcinoma gástrico o hacia linfoma tipo MALT ${ }^{3,4}$.

Se ha postulado que algunos genes de H. pylori, expresados diferencialmente entre cepas, pueden ser usados como marcadores de virulencia ${ }^{8,9}$. Varios estudios sugieren que los factores de adherencia de la bacteria pueden contribuir a su tropismo específico y patogenicidad en el epitelio gástrico humano ${ }^{6,10}$. La adhesina BabA de H. pylori codificada por el gen $b a b A 2$ le permite unirse a antígenos de grupo sanguíneo Lewis b presentes en las células epiteliales gástricas humanas ${ }^{11,12}$. Se ha reportado que la presencia del gen $b a b A 2$ está correlacionada significativamente con el desarrollo de úlcera duodenal y adenocarcinoma gástrico ${ }^{6,10,13-15}$

Otro gen que ha sido implicado en la virulencia de la bacteria es el gen ice $A$ (inducido por contacto con el epitelio) ${ }^{16}$, del que se conocen dos variantes, iceA1 e iceA2, las cuales no están relacionadas entre sí, pero se encuentran ocupando el mismo locus en el genoma bacteriano $^{17}$. El gen iceAl presenta homología con el gen nlaIIIR de Neisseria lactámica que codifica para una endonucleasa de restricción ${ }^{17}$; no obstante, no se conoce si este gen es funcional o si su producto está involucrado en la colonización del estómago por parte de H. pylori ${ }^{18}$. Únicamente se observa la inducción de iceAl por contacto de la bacteria con el epitelio gástrico, y en algunas poblaciones el genotipo ice $A 1$ se ha asociado con la presentación de úlcera péptica ${ }^{16,19,20}$.

Teniendo en cuenta que en la población colombiana la infección por $H$. pylori es muy frecuente y que la 
presencia de determinados factores de virulencia ha sido relacionada con el desarrollo diferencial de patologías gastro-duodenales; el presente estudio, tuvo como objetivo aportar datos sobre la prevalencia de $H$. pylori y su caracterización en base a los genes de virulencia $b a b A 2$ e ice $A$ en pacientes colombianos con patología gastroduodenal benigna.

\section{Pacientes y Métodos}

Se incluyeron en el estudio 60 pacientes que asistieron a la unidad de endoscopia de un consultorio de gastroenterología de la ciudad de Ibagué, Colombia, entre los meses de febrero de 2006 y marzo de 2007. Todos los individuos que participaron, presentaban un cuadro de dispepsia, se les había indicado la realización de una endoscopia alta y firmaron el formato establecido para la consecución del consentimiento informado. Se excluyeron pacientes con enfermedades cardiovasculares y respiratorias, y aquellos que hubieran recibido antiácido 12 horas antes del procedimiento, bloqueadores de la bomba de protones y $\mathrm{H} 2$ quince días antes de la endoscopia y antimicrobianos durante los tres meses previos a la toma de la muestra. El protocolo de trabajo fue aprobado por los Comités de Ética de las instituciones participantes.

Durante el procedimiento endoscópico, el médico gastroenterólogo tomó una biopsia del antro gástrico, destinado a la realización de las pruebas moleculares y 4 a 6 biopsias para la realización del estudio histopatológico. El análisis histopatológico de las muestras fue realizado en cortes histológicos coloreados con hematoxilinaeosina, de acuerdo con los criterios del sistema Sydney modificado ${ }^{21}$ y la tinción de Giemsa para la visualización de bacilos tipo H. pylori.

Extracción de ADN. Se realizó la extracción de ADN a partir de la biopsia de antro gástrico, usando el kit Aqua Pure Genomic DNA de (Bio-Rad $\left.{ }^{\circledR}\right)$ de acuerdo con las indicaciones del fabricante.

RPC Duplex para la amplificación del gen $16 \mathrm{~S}$ ADNr y babA2 de H. pylori. Para establecer la presencia del microorganismo en biopsias de tejido gástrico y realizar la detección del factor de virulencia BabA, se llevó a cabo la amplificación simultánea de un fragmento de 537 pb del gen $16 S A D N r$ y uno de $831 \mathrm{pb}$ del gen babA2 de $H$. pylori, utilizando los partidores ACT-1 (5'-CTTGCTAGAGTGCTGATTA-3') y ACT2 (5'-TCCCACACTCTAGAATAGT-3') $)^{22}$ y babA2F (5'-AATCCAAAAAGGAGAAAAAGTATGAAA-3') y babA2R (5'-TGTTAGTGATTTCGGTGTAGGACA-3' $)^{6}$, respectivamente. Como control positivo de la amplificación se usó ADN extraído a partir de la cepa de referencia NCTC 11637 de H. pylori y como blanco de reacción se usó agua ultrapura en lugar de ADN. Cada mezcla de reacción se realizó en un volumen final de 25 $\mu \mathrm{L}$ que contenía $50 \mathrm{mM}$ de $\mathrm{KCl}, 20 \mathrm{mM}$ de Tris $\mathrm{HCl} \mathrm{pH}$ $(8,4), 3,0 \mathrm{mM}$ de $\mathrm{MgCl}_{2}, 0,2 \mathrm{mM}$ de cada desoxinucleotido trifosfato (dNTPs), $1 \mathrm{pmol} / \mu \mathrm{L}$ de cada partidor $1,25 \mathrm{U}$ de Taq polimerasa (Invitrogen $($ ), y $60 \mathrm{ng} / \mu \mathrm{L}$ de ADN. El perfil térmico consistió en una denaturación inicial a $94{ }^{\circ} \mathrm{C}$ por 4 minutos; seguida de 37 ciclos de denaturación, anillaje y elongación a $94{ }^{\circ} \mathrm{C}(1 \mathrm{~min}), 56{ }^{\circ} \mathrm{C}(1 \mathrm{~min}) \mathrm{y}$ $72{ }^{\circ} \mathrm{C}(1 \mathrm{~min})$, respectivamente, y 10 minutos a $72{ }^{\circ} \mathrm{C}$ para la elongación final. Las reacciones de amplificación se realizaron usando un termociclador modelo iCycler Thermal Cycler de Bio-Rad.

RPC para la amplificación de los genes iceA1 e iceA2 de H. pylori. Para la amplificación de los genes iceA1 e ice $A 2$ de $H$. pylori se usaron los partidores IceA1F5 (5'-GTGTTTTTAACCAAAGTATC-3') - IceA1R4 (5'-CTATAGCCASTYTCTTTGCA -3') e IceA2F6 (5'-GTTGGGTATATCACAATTTAT-3') - IceA2R5 (5'-TTRCCCTATTTTCTAGTAGGT-3') respectivamente $^{17}$. La reacción se realizó en un volumen final de $25 \mu \mathrm{L}$, que contenía $50 \mathrm{mM}$ de $\mathrm{KCl}, 20 \mathrm{mM}$ de Tris $\mathrm{HCl} \mathrm{pH}(8,4)$, 2,5 $\mathrm{mM}$ de $\mathrm{MgCl}_{2}, 0,2 \mathrm{mM}$ de cada desoxinucleotido trifosfato (dNTPs), $1 \mathrm{pmol} / \mu \mathrm{L}$ de cada partidor, $1,25 \mathrm{U}$ de Taq polimerasa (Invitrogen ${ }^{\circledR}$ ), y $60 \mathrm{ng} / \mu \mathrm{L}$ de ADN. El perfil térmico consistió en una denaturación inicial a 94 ${ }^{\circ} \mathrm{C}$ por 9 minutos, seguida de 40 ciclos de: 30 segundos a $94{ }^{\circ} \mathrm{C}, 45$ segundos a $56{ }^{\circ} \mathrm{C}$ y 45 segundos a $72{ }^{\circ} \mathrm{C}$, con un paso de extensión final a $72^{\circ} \mathrm{C}$ por 5 minutos.

RPC para la amplificación del gen de la IL-1 13 humana. Con el fin de descartar falsos negativos por inhibición de la reacción de RPC, se realizó la amplificación de un fragmento del gen de la IL-1 $\beta$ humana en las muestras con resultados negativos para la amplificación de los genes $16 S A D N r$ e iceA de H. pylori. Se emplearon los partidores (5'-AGAAGCTTCCACCAATACTC-3') y (5'-ACCACCTAGTTGTAAGGAAG-3') ${ }^{23}$ y la reacción se llevó a cabo en un volumen final de $25 \mu \mathrm{L}$, que contenía $50 \mathrm{mM}$ de $\mathrm{KCl}, 20 \mathrm{mM}$ de Tris $\mathrm{HCl} \mathrm{pH}(8,4)$, 3,0 $\mathrm{mM}$ de $\mathrm{MgCl}_{2}, 0,2 \mathrm{mM}$ de cada desoxinucleotido trifosfato (dNTPs), $1 \mathrm{pmol} / \mu \mathrm{L}$ de cada partidor, $1,25 \mathrm{U}$ de Taq polimerasa (Invitrogen $\AA$ ), y $60 \mathrm{ng} / \mu \mathrm{L}$ de ADN. El perfil térmico consistió en una denaturación inicial a $94{ }^{\circ} \mathrm{C}$ por 10 minutos, seguida de 5 ciclos de $94{ }^{\circ} \mathrm{C}$ por 30 segundos, $65^{\circ} \mathrm{C}$ por 30 segundos y $72{ }^{\circ} \mathrm{C}$ por 30 segundos, continuando con 30 ciclos de 30 segundos a $94{ }^{\circ} \mathrm{C}, 30$ segundos a $60{ }^{\circ} \mathrm{C}$ y 30 segundos a $72{ }^{\circ} \mathrm{C}$, y 5 ciclos a $94{ }^{\circ} \mathrm{C}$ por 30 segundos, $55^{\circ} \mathrm{C}$ por 30 segundos y $72{ }^{\circ} \mathrm{C}$ por 30 segundos, con un paso de extensión final a $72{ }^{\circ} \mathrm{C}$ por 7 minutos.

Visualización y análisis de los productos de amplificación. Los productos de amplificación fueron separados 
mediante electroforesis en geles de agarosa. La detección y visualización se realizó con bromuro de etidio bajo luz $\mathrm{UV}$, en un sistema de documentación de geles ChemiDoc system XRS de BioRad®. Para el procesamiento y análisis de las imágenes se utilizó el programa Quantity One de BioRad $\AA$.

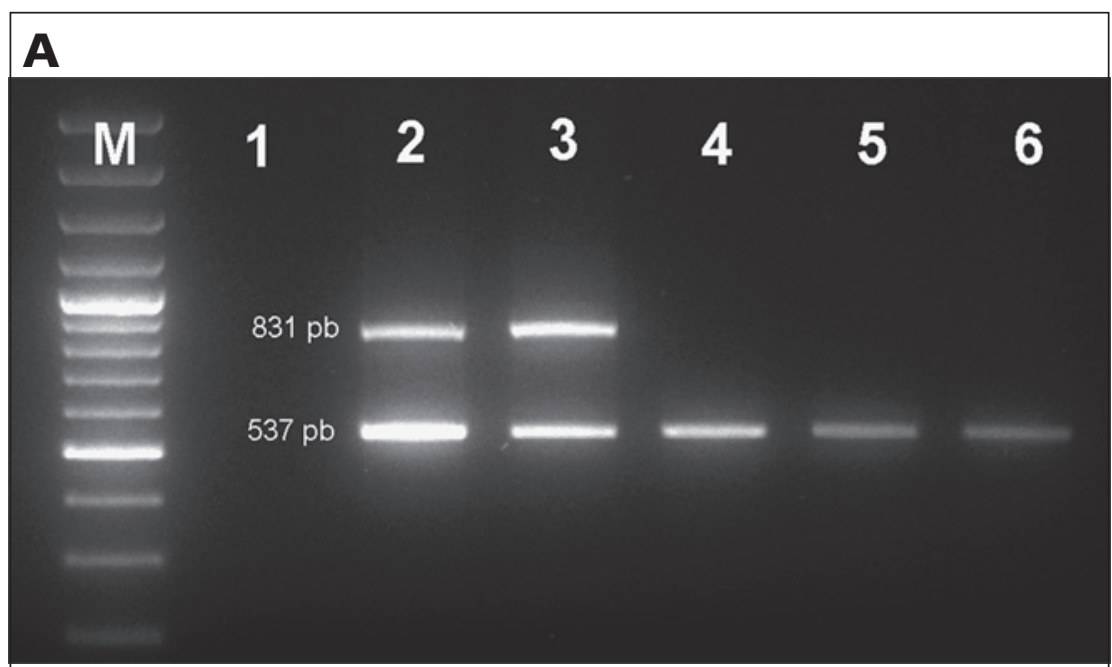

B

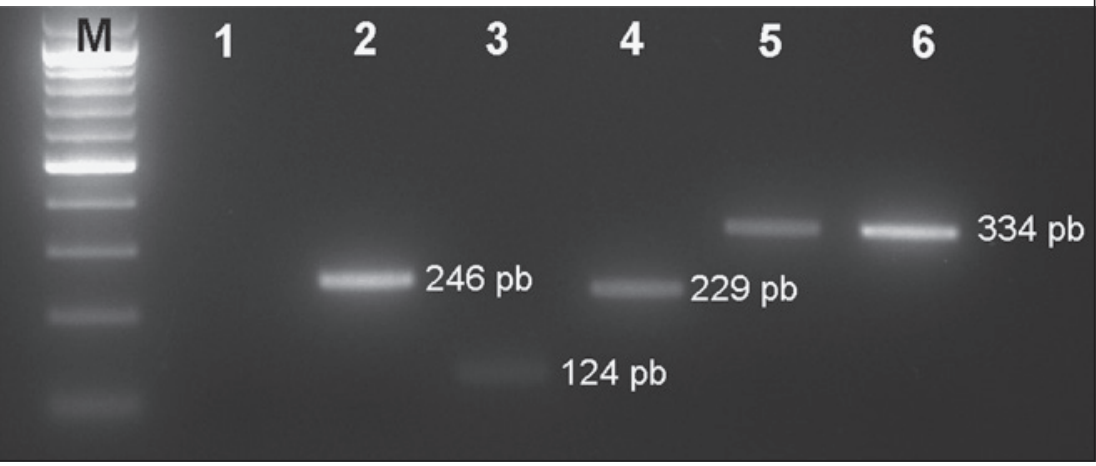

Figura 1. A) Detección del gen $165 \operatorname{ADNr}(537 \mathrm{pb})$ y el gen de virulencia babA2 (831 pb) de H. pylori en muestras de biopsia gástrica. M: Marcador de peso molecular de 100 pb, 1: blanco de reacción, 2 : cepa NCTC 11637 de H. pylori, 3: muestra positiva para 16S ADNry babA2, 4-6: muestras positivas para 16S ADNr. B). Detección de los genes iceA1 (246 pb) e iceA2 (124, 229 o 334 pb) de H. pylori en muestras de biopsia gástrica. M: Marcador de peso molecular de 100 pb, 1: blanco de reacción, 2 muestra positiva para iceA1, 3-5: muestras positivas para iceA2 y 6: cepa NCTC 11637 de H. pylori.
Análisis estadísticos. Para el análisis estadístico se empleó el programa SPSS versión 17.0. Se utilizó un modelo de regresión multinomial para establecer si la presencia de los genes estudiados permitía predecir alguno de los estados de la enfermedad gástrica. Se consideró estadísticamente significativa una probabilidad $(\mathrm{p})<0,05$.

\section{Resultados}

Se analizaron biopsias gástricas provenientes de 60 pacientes, 28 hombres y 32 mujeres, con edad promedio de 54,2 \pm 15,5 años. La evaluación histopatológica reveló que dos pacientes $(3 \%)$ no presentaban alteraciones de la mucosa gástrica y se diagnosticó gastritis no atrófica en 58 pacientes $(97 \%)$. Los hallazgos endoscópicos indicaron que 10 de los 58 pacientes con gastritis no atrófica presentaron diagnóstico de úlcera (Tabla 1).

La presencia de H. pylori se detectó en 38 de las 60 muestras analizadas $(63,3 \%)$ por medio de la amplificación de un fragmento de $537 \mathrm{pb}$ del gen $16 \mathrm{~S} \mathrm{ADNr}$ especie-específico (Figura 1A). Acoplada a esta reacción de RPC, se realizó la detección del factor de virulencia BabA, mediante la amplificación de un fragmento del gen babA2. El producto esperado de esta reacción corresponde a un fragmento de $831 \mathrm{pb}$ (Figura 1A), el cual fue observado en 7 de las 38 muestras $H$. pylori positivas. No se observo amplificación del gen $b a b A 2$ en las biopsias con resultados negativos para la amplificación del gen $16 \mathrm{~S}$ $A D N r$ (Tabla 1).

La presencia de los genes iceA1 e ice $A 2$ fue evaluada en todas las muestras incluidas en este estudio. El producto esperado de estas reacciones corresponde a un fragmento de $246 \mathrm{pb}$ para el gen iceA1 y 124, 229, 334 o $439 \mathrm{pb}$ para el gen iceA2. En 40 de las 60 muestras evaluadas $(66,6 \%)$, se observó amplificación de uno o ambos alelos del gen iceA. De estos 40 casos, 14 pacientes eran portadores de cepas con genotipo iceA1 y 19 pacientes portaban cepas ice $A 2$, las cuales se distribuyeron, según el tamaño del producto de amplificación, de la siguiente forma: (1/19) 124 pb, (9/19) 229 pb y (9/19) 334 pb (Figura 1B). En 7 pacientes se observó la amplificación de ambos genes (iceA1 e iceA2) o la de variantes del gen iceA2, lo cual

Tabla 1. Frecuencia de los genes 165 ADNr, iceA y babA2 de H. pylori en la población de estudio, según el diagnóstico histológico

\begin{tabular}{|c|c|c|c|c|c|c|}
\hline Diagnóstico histológico & n $/ \%$ & $16 S$ ADNr & iceA1 & iceA2 & Coinfección & babA2 \\
\hline Ausencia de patología & $2 \quad(3)$ & 2 & 2 & 0 & 0 & 0 \\
\hline Gastritis & $48 \quad(80)$ & 29 & 10 & 16 & 4 & 6 \\
\hline Gastritis y úlcera & $10 \quad(17)$ & 7 & 2 & 3 & 3 & 1 \\
\hline Total & $60(100)$ & 38 & 14 & 19 & 7 & 7 \\
\hline
\end{tabular}


es un indicativo de la presencia de más de una cepa de $H$. pylori en la muestra de biopsia. En 20 de las 60 muestras analizadas no se observó amplificación de iceA (Tabla 1).

Es importante resaltar que al comparar los resultados obtenidos para la detección de H. pylori por medio de la identificación del bacilo en tinciones histológicas con los de las pruebas moleculares de amplificación del gen $16 \mathrm{~S}$ $A D N r$ y la tipificación del locus iceA, se observó concordancia entre los tres métodos en 45 de las 60 muestras. En las 15 muestras restantes se observa concordancia entre dos de los tres métodos (Figura 2).

Teniendo en cuenta que se ha reportado la existencia de factores inhibidores de la RPC en biopsias gástricas ${ }^{24}$, se realizó la amplificación del gen de la IL-1 $\beta$ humana con el fin de descartar falsos negativos. El producto esperado de esta reacción se encontró presente en todas las muestras analizadas, lo que indica la ausencia de factores inhibidores.

Se procedió a determinar si los genes babA2 e ice $A$ de $H$. pylori podían estar relacionados con la presentación de alguna de las patologías gástricas. Los valores de $\mathrm{p}$ siempre fueron mayores a 0,05 (datos no mostrados), lo que indica que no se encontró relación estadísticamente significativa entre la presencia de estos factores de virulencia y la presentación de úlcera en la población analizada.

\section{Discusión}

El diagnóstico de la infección por $H$. pylori es de gran importancia, dado que su presencia ha sido asociada con el desarrollo de algunas enfermedades gastro-duodenales ${ }^{3,4,25}$. Existen diferentes pruebas para diagnosticar la infección por $H$. pylori, cada una de ellas con ventajas y desventajas $^{5}$. Los métodos de detección se han dividido tradicionalmente en métodos invasores y no invasores, según si requieren o no de una endoscopia con toma de biopsia ${ }^{4,25}$. En nuestro medio, el diagnóstico se basa principalmente en la detección del microorganismo en preparados histológicos y en la prueba de ureasa en especímenes de biopsia. El cultivo de H. pylori no se realiza habitualmente en el diagnóstico inicial de la infección; sin embargo, se recomienda su uso cuando ha ocurrido una falla terapéutica y no se ha logrado su erradicación ${ }^{3,5,25}$.

La reacción de RPC permite confirmar el diagnóstico de la infección, mediante la detección y amplificación de un fragmento de ADN especifico de $H$. pylori ${ }^{4,25}$. Dada la especificidad de los partidores y la capacidad de la técnica de amplificar exponencialmente el ADN blanco presente en una muestra, se ha reportado que presenta una alta especificidad y sensibilidad, ofreciendo un diagnostico rápido y confiable de la infección, que es de gran utilidad en la detección de microorganismos de difícil cultivo como es el caso del H. pylori. Adicionalmente, la aplicación de esta técnica se extiende a la detección

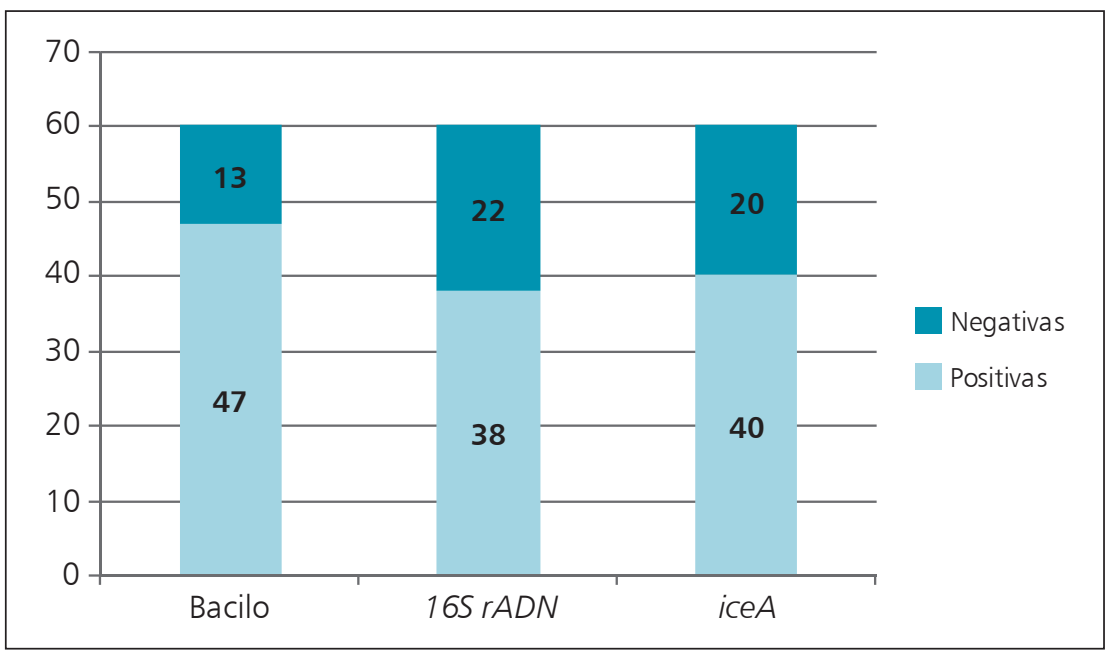

Figura 2. Resultados de la detección de H. pylori por medio de la identificación del bacilo en tinciones histológicas y las pruebas moleculares de amplificación del gen 165 ADNry tipificación del locus iceA.

de factores genéticos responsables de la virulencia y en la evaluación de mutaciones específicas asociadas a la resistencia a antimicrobianos ${ }^{26,27}$.

En este estudio se observó una prevalencia de la infección de 78,3\% por medio de la detección de bacilos tipo Helicobacter y 63,3\% mediante la aplicación de la técnica de RPC para la amplificación de un fragmento del gen $16 \mathrm{~S}$ $A D N r$ de H. pylori (Figura 2). Bravo y cols, analizaron reportes histológicos de hospitales de diferentes ciudades capitales de Colombia durante 1997, y encontraron una prevalencia de la infección por $H$. pylori en biopsias de estómago de $69,1 \%$, reportando una prevalencia para la ciudad de Ibagué de $59,5 \%{ }^{28}$. Estudios similares han reportado una alta prevalencia de la infección en Colombia ${ }^{29}$ y en otros países de Latinoamérica ${ }^{8,30}$.

Al comparar la detección de $H$. pylori a través de la identificación del bacilo con la detección molecular a través de la amplificación de un fragmento del gen $16 \mathrm{~S}$ $A D N r$ especie-específico, se observan algunas discrepancias en cuanto a que en 12 pacientes se detectó la presencia del bacilo por medio de la tinción histológica de Giemsa pero el resultado de la amplificación del gen $16 S A D N r$ fue negativo; y en dos pacientes se realizó la detección del gen $16 S A D N r$ pero la identificación del bacilo fue negativa (Figura 2). El índice kappa entre estas dos pruebas fue de 0,41 lo cual indica una concordancia moderada. Otros estudios también han reportado mediante tinción histológica la presencia de bacilos en muestras con resultados negativos de la RPC específica de H. pylori ${ }^{30}$.

Teniendo en cuenta que el locus iceA, está siempre presente en $H$. pylori, la detección del mismo, a través de la amplificación de los genes iceA1 e iceA2 puede 
ser usada para la detección de este microorganismo. Al calcular el índice de concordancia kappa entre la detección de $H$. pylori a través de la tipificación del locus ice $A$ y la detección del gen $16 S A D N r$, se obtuvo un valor de 0,94 , indicativo de una concordancia excelente entre los blancos moleculares. Únicamente se presentó discrepancia en dos pacientes, en quienes se observó la presencia del gen iceA2, pero no hubo amplificación del gen $16 \mathrm{~S} A D N r$. En estas dos muestras la identificación del bacilo a través de la tinción de Giemsa fue positiva (Figura 2). Asimismo se evalúo la concordancia entre la detección de $H$. pylori a través de la detección del locus ice $A$ y la identificación microscópica del bacilo. En este último caso en índice kappa fue de 0,51 indicativo de una concordancia moderada entre los dos métodos.

Es posible que la discrepancia entre la detección molecular (16S ADNr e iceA) y la identificación del bacilo en cortes histológicos, se deba a diferencias en el lugar de toma de la biopsia y a que los humanos pueden estar colonizados por otras especies de Helicobacter v.gr., $H$. pullorum y $H$. helmannii. Teniendo en cuenta que la colonización de la mucosa gástrica por parte de $H$. pylori es en forma de "parches" 31 y que en las pruebas moleculares se trabajó siempre con una biopsia de antro gástrico (zona de colonización preferencial del microorganismo debido a la ausencia de células parietales ${ }^{32}$ ) y para la realización del estudio histopatológico se obtuvieron de 4 a 6 biopsias según criterio médico; las muestras no necesariamente provenían de la misma región del estómago, motivo que podría explicar al menos en parte la discrepancia entre la detección molecular y la histología. Adicionalmente, aunque la histología ha sido considerada por algunos autores el estándar de oro para el diagnóstico de la infección por H. pylori ${ }^{25}$, es importante resaltar que H. pylori y H. heilmannii (microorganismo que puede infectar la mucosa gástrica de humanos) presentan características morfológicas altamente similares ${ }^{3}$, hecho que dificulta la diferenciación entre estas dos especies de Helicobacter. La caracterización de las cepas de H. pylori reveló que sólo 7 pacientes eran portadores de cepas babA2 positivo. Estudios en otras poblaciones muestran variaciones significativas en la frecuencia de presentación del genotipo babA2. En Japón ${ }^{33}$ y China ${ }^{10}$ se ha encontrado una alta frecuencia de cepas babA2 positivas $(84,9$ y $79,8 \%$ respectivamente), por el contrario los resultados en poblaciones de Italia $(36 \%)^{15}$, Corea $(36,1 \%)^{34}$, Brasil $(46,1 \%)^{13}$ y Bogotá-Colombia $(48 \%)^{7}$ revelan una proporción más baja de cepas babA2 positivas. Es posible que la baja proporción de cepas $b a b A 2$ positivas se deba a que la población de estudio corresponde a pacientes con patología gástrica benigna y BabA es un factor de virulencia que se ha asociado con la presentación de patologías gástricas graves.

Los genotipos iceA1 e iceA2 fueron detectados en 35 y $47,5 \%$ de las cepas positivas para la presencia del locus ice $A$, respectivamente. Adicionalmente, en $17,5 \%$ de los casos, se encontró infección por más de una cepa de $H$. pylori, lo cual fue evidenciado por la amplificación de ambos genes (iceA1 e iceA2) o variantes del gen iceA2. Estos resultados son muy similares a los reportados anteriormente para cepas colombianas (ice $A 1=40,9 \%$, ice $A 2=42,6 \%$, infección múltiple $=16,5 \%)^{35}$; sin embargo, la caracterización de este locus en otras regiones geográficas muestra variación en la distribución de los genotipos $^{23,36,37}$.

Diversas líneas de evidencia sugieren que los genes babA2 e iceA1 de H. pylori, expresados diferencialmente entre cepas, pueden ser usados como marcadores de virulencia de la bacteria y pueden jugar un papel importante en el desarrollo diferencial de las patologías gástricas. En este estudio, la presencia del gen $b a b A 2$ no se relacionó con la presentación de úlcera. Estos resultados son similares a los reportados en poblaciones de Colombia ${ }^{7}$, Japón $^{33}$, Corea $^{34}$, y Taiwán ${ }^{38}$, donde no se encontró asociación entre la presencia del gen $b a b A 2$ y el resultado clínico de la infección. Asimismo, la presencia del gen iceAl ha sido asociada con la presentación de úlcera péptica y cáncer gástrico $^{16,19,20}$. Los resultados de este estudio no indican relación entre la presencia de los genes iceA1 o iceA2 y el resultado clínico de la infección.

Teniendo en cuenta que diferentes estudios no han encontrado correlación entre la presencia de estos genes y la gravedad de las manifestaciones clínicas, se hace necesario determinar en nuestro medio la utilidad de estos genes como marcadores de virulencia de la bacteria. En este sentido, estudios de genotipificación del microorganismo, así como la caracterización de factores genéticos del hospedero, en pacientes con patologías gástricas leves e infección activa por $H$. pylori son de gran importancia dado que un porcentaje significativo podría desarrollar alguna patología de mayor gravedad.

Agradecimientos. A la Facultad de Ciencias de la Universidad de los Andes, por haber financiado este proyecto. A los estudiantes de medicina de la Universidad del Tolima que participaron en la consecución de las muestras. Al laboratorio de Inmunología del Instituto Nacional de Cancerología por suministrar la cepa de referencia de H. pylori usada como control en el presente estudio.

\section{Resumen}

Objetivo: El presente estudio investiga la presencia de Helicobacter pylori en pacientes de una población colombiana con enfermedad gastro-duodenal benigna y realiza su genotipificación usando como blanco los genes babA2 e iceA. Pacientes y Métodos: Se analizaron 
biopsias gástricas de 60 pacientes usando histopatología y RPC. Resultados: La presencia de H. pylori se demostró en $78,3 \%, 63,3 \%$ y $66,7 \%$ de los pacientes, mediante tinción de Giemsa, amplificación del gen $16 S A D N r$ y del locus iceA, respectivamente. Helicobacter pylori babA2 positivo se encontró infectando 7 pacientes y de las 40 muestras positivas para la presencia del locus ice $A$,
$35 \%$ eran iceA1, $47.5 \%$ ice $A 2$ y en $17,5 \%$ se evidenció infección múltiple. Se aportan datos sobre la prevalencia de $H$. pylori encontrando una baja proporción de casos babA2 positivos y una distribución de los genotipos ice $A$ acorde con datos reportados previamente. La presencia de los genes babA2 e iceA no se encontró asociada con la presentación de úlcera.

\section{Referencias}

1.- Marshall B J, Warren J R. Unidentified curved bacilli in the stomach of patients with gastritis and peptic ulceration. Lancet 1984; 1: 1311-5.

2.- Blaser M J, Atherton J C. Helicobacter pylori persistence: biology and disease. J Clin Invest 2004; 113: 321-33.

3.- Suerbaum S, Michetti P. Helicobacter pylori Infection. N Engl J Med 2002; 347: 1175-86.

4.- Rivas-Traverso F, Hernández F. Helicobacter pylori: Factores de virulencia, patología y diagnóstico. Rev Biomed 2000; 11: 187-205.

5.- Logan R P, Walker M M. Epidemiology and diagnosis of Helicobacter pylori infection. $\mathrm{Br}$ Med J 2001; 323: 920-2.

6.- Gerhard M, Lehn N, Neumayer N, Boren T, Rad R, Schepp W, et al. Clinical relevance of the Helicobacter pylori gene for blood-group antigen-binding adhesin. Proc Natl Acad Sci 1999; 96: 12778-83.

7.- Quiroga A, Cittelly D, Bravo M. Frecuencia de los genotipos babA2, oipA y cagE de Helicobacter pylori en pacientes colombianos con enfermedades gastroduodenales. Biomédica 2005; 25: 325-34.

8.- Castillo G, Mazarí M, López Y. Helicobacter pylori: Focus on CagA and VacA major virulence factors. Salud Publica Mex 2004; 46: 538-48.

9.- Boren T, Normark S, Falk P. Helicobacter pylori: molecular basis for host recognition and bacterial adherence. Trends Microbiol 1994; 2 : 221-8.

10.- Yu J, Leung W K, Go M Y, Chan M C, To K F, Ng E K, et al. Relationship between Helicobacter pylori babA2 status with gastric epithelial cell turnover and premalignant gastric lesions. Gut 2002; 51: 480-4.

11.- Falk P G, Roth K A, Boren T, Westblom U, Gordon J I, Normark S. An in vitro adherence assay reveals that Helicobacter pylori exhibits cell lineage-specific tropism in the human gastric epithelium. Proc Natl Acad Sci 1993; 90: 2035-9.

12.- Falk P G, Bry L, Holgersson J, Gordon J I. Expression of a human alpha-1,3/4-fucosyltransferase in the pit cell lineage of $\mathrm{FVB} / \mathrm{N}$ mouse stomach results in production of Leb-containing glycoconjugates: a potential transgenic mouse model for studying Heli- cobacter pylori infection. Proc Natl Acad Sci 1995; 92: 1515-9.

13.- Oliveira A G, Santos A, Guerra J B, Rocha G A, Rocha A M, Oliveira C A, et al. babA2- and cagA-positive Helicobacter pylori strains are associated with duodenal ulcer and gastric carcinoma in Brazil. J Clin Microbiol 2003; 41: 3964-6.

14.- Yamaoka Y, Souchek J, Odenbreit S, Haas R, Arnqvist A, Boren T, et al. Discrimination between cases of duodenal ulcer and gastritis on the basis of putative virulence factors of Helicobacter pylori. J Clin Microbiol 2002; 40: 2244-6.

15.- Zambon C F, Navaglia F, Basso D, Rugge M, Plebani M. Helicobacter pylori babA2, cagA, and $\mathrm{s} 1 \mathrm{vacA}$ genes work synergistically in causing intestinal metaplasia. J Clin Pathol 2003; 56: 287-91.

16.- Peek R M, Thompson S A, Donahue J P, Tham K T, Atherton J C, Blaser M J, et al. Adherence to gastric epithelial cells induces expression of a Helicobacter pylori gene, iceA, that is associated with clinical outcome. Proc Assoc Am Physicians 1998; 110: 531-44.

17.- Figueiredo C, Quint W G, Sanna R, Sablon E, Donahue J P, Xu Q, et al. Genetic organization and heterogeneity of the iceA locus of Helicobacter pylori. Gene 2000; 246: 59-68.

18.- Xu Q, Morgan R D, Roberts R J, Xu S Y, van Doorn L J, Donahue J P, et al. Functional analysis of iceAl, a CATG-recognizing restriction endonuclease gene in Helicobacter pylori. Nucleic Acids Res 2002; 30: 3839-47.

19.- van Doorn L J, Figueiredo C, Sanna R, Plaisier A, Schneeberger P, de Boer W, et al. Clinical relevance of the $\operatorname{cag} A, v a c A$, and ice $A$ status of Helicobacter pylori. Gastroenterology 1998; 115: 58-66.

20.- Wu C C, Chou P Y, Hu C T, Liu Z C, Lin C Y, Tseng Y H, et al. Clinical relevance of the $v a c A$, ice A, cagA, and flaA genes of Helicobacter pylori strains isolated in Eastern Taiwan. J Clin Microbiol 2005; 43: 2913-15.

21.- Dixon M F, Genta R M, Yardley J H. Classification and grading of gastritis. The updated Sydney System. International Workshop on the Histopathology of Gastritis, Houston 1994. Am J Surg Pathol 1996; 20: 1161-81.

22.- Thoreson A C, Borre M B, Andersen L P,
Elsborg L, Holck S, Conway P, et al. Development of a PCR-based technique for detection of Helicobacter pylori. FEMS Immunol Med Microbiol 1995; 10: 325-33.

23.- Zeng Z, Hu J, Hu S, Pang R, Chen M, Sung J. Association of interleukin 1B gene polymorphism and gastric cancers in high and low prevalence regions in China. Gut 2003; 52 : 1684-9.

24.- Thoreson A C E, Borre M L P, Andersen F, Jorgensen S, Kiilerich J, Scheibel, et al. Helicobacter pylori detection in human biopsies: a competitive PCR assay with internal control reveals false results. FEMS Immunol Med Microbiol 1999; 24: 201-8.

25.- Hardin F J, Wright R A. Helicobacter pylori: Review and update. Hosp Physician 2002; 38: 23-31.

26.- Premoli G, González A, Millán-Mendoza, Percoco T, Vielmas A. Diagnóstico de Helicobacter pylori mediante la reacción en cadena de la polimerasa. Rev Cubana Med Trop 2004; 56 : 85-90

27.- Mégraud F, Lehours P. Helicobacter pylori detection and antimicrobial susceptibility testing. Clin Microbiol Rev 2007; 20: 280-322.

28.- Bravo LE, Cortés A, Carrascal E, Jaramillo R, García L S, Bravo P E, et al. Helicobacter pylo$r i$ : patología y prevalencia en biopsias gástricas en Colombia. Colomb Med 2003; 34: 124-31.

29.- Martínez J D, Henao S C, Granados C. La gastritis crónica atrófica corporal y la edad. Rev Col Gastroenterol 2007; 22: 17-22.

30.- Araya J C, Anabalón L, Roa I, Bravo M, Villaseca M A, Guzmán P, et al. Relación de la genotipificación de Helicobacter pylori con la forma e intensidad de la gastritis en población adulta portadora de patología gástrica benigna. Rev Méd Chile 2004; 132: 1345-54.

31.- Bayerdörffer E, Oertel H, Lehn N, Kasper G, Mannes G A, Sauerbruch T, et al. Topographic association between active gastritis and Campylobacter pylori colonization. J Clin Pathol 1989; 42: 834-9.

32.- Lundin A, Bjorkholm B, Kupershmidt I, Unemo M, Nilsson P, Andersson D I, et al. Slow genetic divergence of Helicobacter pylori strains during long-term colonization. Infect Immun 2005; 73: 4818-22.

33.- Mizushima T, Sugiyama T, Komatsu Y, 
Ishizuka J, Kato M, Asaka M. Clinical relevance of the babA2 genotype of Helicobacter pylori in Japanese clinical isolates. J Clin Microbiol 2001; 39: 2463-5.

34.- Kim S Y, Woo C W, Lee Y M, Son B R, Kim J W, Chae H B, et al. Genotyping CagA, VacA subtype, IceA1, and BabA of Helicobacter pylori isolates from Korean patients, and their association with gastroduodenal diseases. J Korean Med Sci 2001; 16: 579-84.
35.- Cittelly D M, Huertas M G, Martínez J D, Oliveros R, Posso H, Bravo M M, et al. Los genotipos de Helicobacter pylori en gastritis no atrófica difieren de los encontrados en úlcera péptica, lesiones premalignas y cáncer gástrico en Colombia. Rev Méd Chile 2002; 130: 143-51.

36.- Ashour A A, Birchal G, Nogueira E, Ribeiro V, Queiroz D M, Prazeres P, et al. iceA Genotypes of Helicobacter pylori strains isolated from Brazilian children and adults. J Clin Microbiol
2001; 39: 1746-50.

37. Nimri L F, Matalka I, Hani K B, Ibrahim M. Helicobacter pylori genotypes identified in gastric biopsy specimens from Jordanian patients. BMC Gastroenterol 2006; 6: 27.

38. Lai C H, Kuo C H, Chen Y C, Chao F Y, Poon S K, Chang C S, et al. High prevalence of cagA- and babA2-positive Helicobacter pylori clinical isolates in Taiwan. J Clin Microbiol 2002; 40: 3860-62. 